Ciudad y territorio 


\section{Cerrando espacios, abriendo brechas. Urbanizaciones cerradas en San Rafael de Escazú, Costa Rica}

Lic. Alejandro Alvarado Alcázar, Sociólogo

Invitado nacional

Instituto de Investigaciones Sociales, Universidad de Costa Rica

al.alvacazar@gmail.com

Recibido: setiembre del 2015

Aceptado: mayo del 2016

Ciudad y territorio

Investigación 


\section{Resumen}

El presente artículo estudia el fenómeno de las urbanizaciones cerradas en el distrito de San Rafael de Escazú, Costa Rica. La tesis central es que las urbanizaciones cerradas producen cambios en la urbanidad mediante la introducción de transformaciones socio-espaciales manifiestas en la materialidad de las urbanizaciones cerradas, las funciones de estas y el tipo de relaciones sociales que sus residentes mantienen entre sí y con su entorno cercano. El artículo cierra sugiriendo explorar la noción de "urbanidad privada" como una forma de entender la producción de espacio promovida desde los intereses privados expresados en la construcción de conjuntos inmobiliarios cerrados, de los cuales las urbanizaciones cerradas son una de sus manifestaciones más visibles. Los resultados presentados en este ensayo, son producto de la investigación titulada "Urbanizaciones cerradas en Costa Rica: transformaciones socio-espaciales en la urbanidad y segregación socio-residencial en el distrito de San Rafael de Escazú (1990-2012)".

Palabras clave: inseguridad; urbanizaciones cerradas; urbanismo neoliberal.

\section{Abstract}

This paper examines the phenomenon of gated communities in Costa Rica, specifically in the district of San Rafael, Escazú. The central thesis is that gated communities to changes in urbanity by introducing socio-spatial transformations evident in the materiality of gated communities, the functions of these communities and the type of social relations that its residents have with one another and with their immediate environment. The article suggests exploring the notion of "private urbanity" as a way to understand the production of space promoted by private interests and expressed in the construction of closed real estate developments, most visibly manifested by gated communities.. The results presented are the product of research entitled "Urbanizaciones cerradas en Costa Rica: transformaciones socio-espaciales en la urbanidady segregación socio- residencial en el distrito de San Rafael de Escazú (1990-2012)”.

Keywords: insecurity; gated communities; neoliberal urbanism. 


\title{
Cerrando espacios, abriendo brechas: Urbanizaciones cerradas en San Rafael de Escazú, Costa Rica
}

\author{
Alejandro Alvarado Alcázar ${ }^{1}$
}

\section{Introducción}

Entre los cambios escenificados durante las últimas décadas en el sistema urbano costarricense, las nuevas modalidades residenciales se colocan como uno de los fundamentales. Desde los años 1990, y con una intensidad inusitada durante la primera década de este siglo, los condominios o urbanizaciones cerradas ${ }^{2}$ se transformaron en la modalidad dominante entre los sectores de clase media-alta y alta en Costa Rica. En este artículo se propone estudiar el desarrollo de esta forma urbana, tomando como base el caso de San Rafael de Escazú, en la zona Oeste de San José.

Para esto se desarrollará primero un resumido marco teórico, centrándose en los principales procesos de cambio en la ciudad durante el último tiempo, tomando como punto de partida el llamado urbanismo neoliberal (Theodore et al, 2009) y la discusión sobre la (in)seguridad. Posteriormente, se presenta una síntesis metodológica en la cual se definen las tres dimensiones de análisis consideradas: formas urbanas, funciones urbanas y relaciones sociales. El artículo continúa con un análisis de los modos cómo se manifiestan dichas dimensiones en la dinámica de las urbanizaciones cerradas y cierra con una serie de conclusiones sobre este fenómeno.

Las urbanizaciones cerradas se definen como una modalidad residencial fundada en la separación de un espacio mediante la colocación de barreras físicas, la cual cuenta con espacios para el uso común de sus residentes y una forma de organización propia (Janoschka y Glasze, 2003)3. Para el caso de este trabajo, se ha considerado únicamente la modalidad residencial de tipo horizontal considerada como la predominante en el distrito de estudio. Si bien se identificaron un conjunto de desarrollos verticales los cuales han tendido a crecer en número durante los últimos años, se tomó la decisión

\footnotetext{
$1 \quad$ Licenciado en Sociología de la Universidad de Costa Rica. Actualmente labora como investigador en el Instituto de Investigaciones Sociales de la misma universidad donde coordina un proyecto de investigación sobre seguimiento de las acciones colectivas. Entre sus intereses de investigación se encuentran los movimientos sociales, el conflicto social y las temáticas relacionadas con el espacio y el territorio.

2 Pese a los múltiples términos usados para nombrar esta forma urbana en América Latina y el mundo, en este caso se utilizará la denominación genérica "urbanizaciones cerradas" (en inglés "gated communities") para efectos de descripción. En el caso costarricense, en la legislación que regula esta materia y en el lenguaje de uso cotidiano se les denomina como condominios, sean verticales u horizontales.

En este artículo se consideran exclusivamente las urbanizaciones cerradas de carácter horizontal, las cuales en el caso costarricense fueron las primeras en desarrollarse y son las que guardan relación con los estudios desarrollados en otros lugares de América Latina y el mundo.
} 
de trabajar únicamente con los desarrollos horizontales considerando que existen diferencias en los perfiles socio-demográficos de los habitantes de cada uno y en relación con las categorías de análisis de este trabajo.

La tesis central de este texto es que las urbanizaciones cerradas producen cambios en la urbanidad, esto es en el conjunto de propiedades de un espacio (Thuillier, 2005) mediante la introducción de transformaciones socio-espaciales manifiestas en la materialidad de las urbanizaciones cerradas, las funciones de estas y el tipo de relaciones sociales que sus residentes mantienen entre sí y con su entorno cercano. En este sentido, este tipo de forma urbana ha sido uno de los principales motores de cambio en el espacio urbano durante los últimos treinta años, de la mano con la creciente centralidad de los intereses privados en la conducción de la política urbana en Costa Rica. Los cambios mencionados han ocasionado un debilitamiento en el tejido urbano de la ciudad, en tanto han contribuido en el ensanchamiento de la distancia social entre "ricos" y "pobres" y en la consolidación de la segregación socio-espacial.

\section{Urbanismo Neoliberal y el discurso de la (In)seguridad}

Por su ubicación subordinada en el mercado mundial, los procesos de cambios estructural en Costa Rica, como en el resto de América Latina, supusieron transformaciones en casi todos los niveles -i.e. económico, político, social, etc.--. Desde la década de los años 1980, se inició un proceso de neoliberalización de la estructura económica costarricense, el cual se reflejó en un conjunto de cambios en el sistema urbano, incluidos la constitución de un mercado de suelos, la desregulación de las políticas urbanas, tanto en el nivel nacional como en el nivel local y, de la mano de lo anterior, la creciente participación de actores privados, particularmente desarrolladores y promotores inmobiliarios y la banca, en la definición de los rumbos de la ciudad.

Progresivamente, el Estado cedió terreno a los actores privados en la orientación de la política urbana, limitando sus funciones como un sostén técnico-jurídico para (des)regular el mercado inmobiliario en Costa Rica. De esta manera, se instalaba como dominante el "nuevo urbanismo" (Smith, 2005) también denominado como "urbanismo neoliberal" (Theodore et al., 2009) bajo el cual la dirección de los procesos de (re)estructuración urbana se definían en función de los intereses de los capitales privados y de la obtención de beneficios económicos4.

\footnotetext{
4 Tal como plantea Janoschka (2011) una de las características de la producción de lo que él denomina la "ciudad neoliberal" son las nuevas reglas (económico-políticas) fijadas para hacer de la ciudad una mercancía. Estas reglas se orientan fundamentalmente hacia beneficiar los intereses capitalistas mediante la liberalización y la privatización (p. 121).
} 
La creencia de que el mercado se auto-regula y tiene la capacidad por sí solo de guiar el desarrollo urbano se impuso en el plano ideológico, mientras en el plano político se mostraba necesaria la acción estatal para generar las condiciones de posibilidad para el despliegue de los intereses de los capitales privados ${ }^{5}$. En esta dirección, uno de los terrenos donde se evidenció dicha "co-existencia" fue en el mercado de la vivienda. Desde finales de los años 1980, el Estado costarricense prácticamente abandonó toda política de vivienda orientada a dotar de una solución a los sectores de clase media y clase baja, mientras estimulaba la acción privada en la dotación de vivienda para los nuevos sectores medios-altos y altos en formación, como consecuencia de la nueva economía centrada en la tercerización ${ }^{6}$.

Esto supuso la constitución de un mercado de vivienda regulado fundamentalmente por el fin de lucro de las empresas inmobiliarias y los altos niveles de especulación sobre el suelo. Para hacer esto posible, uno de los procesos consustanciales fue la identificación de lugares con una potencial (alta) rentabilidad de suelo, siendo las direcciones Este y Oeste, escasamente urbanizadas hasta ese momento, las de mayor potencial de valorización.

Una forma particular de suburbanización ${ }^{7}$ se impuso desde los años 1990, como un movimiento hacia las márgenes periféricas de la ciudad, sobre todo, como se ha dicho, hacia estos dos polos de la ciudad, los cuales se constituyeron como direcciones de "éxito" para las llamadas nuevas clases medias (Araya, 2010). Un conjunto de cambios en la condición de estos lugares se expresaron en un proceso de tránsito desde una dinámica fundamentalmente rural hacia una de carácter urbano o rural-urbano como la denominó Thuillier (2005) para otras ciudades de América Latina. La constitución de un mercado de suelos y la presión inmobiliaria sobre estos, posibilitó un intenso proceso de urbanización por parte de actores privados de escala nacional y regional que definieron los términos de dicho desarrollo.

\footnotetext{
5 Tal como han señalado distintos autores, entre ellos Harvey (2007) y Theodore et al (2009), existen diferencias sustanciales entre los postulados ideológicos neoliberales y su implementación política concreta. Difícilmente, el neoliberalismo se ha impuesto en su forma pura debido a un conjunto de trayectorias institucionales y marcos regulatorios previos que necesariamente lo obligan a situarse contextualmente. Esto es lo que Theodore et al (2009) ha denominado como "neoliberalismo realmente existente" (p. 2-3).

6 La década de los años 1980 representó un período de mucha tensión social relacionada con las demandas de solución de vivienda sobre todo de sectores urbano-marginados. En dicho período, el Estado ensayó distintas estrategias políticas para enfrentar los conflictos, desde la violencia estatal, tanto física como simbólica, hasta el recurso clientelar. Para un análisis general de estos procesos, ver Mora, 2007. Y para un estudio de caso, ver Chacón y Zuñiga (2014).

$7 \quad$ Cabe señalar que en Costa Rica, este proceso de suburbanización es tardío en comparación con otros países de América Latina y el mundo. Si bien, en casos como el de Escazú, antes de 1990, existían ciertos lugares destinados para uso residencial de las clases altas "históricas", no se trataba de un uso sistemático. Asimismo, tanto hacia el Este como hacia el Oeste, predominaban otros usos de carácter rural como la tierra para la producción de café y el conjunto de dinámicas sociales que esto implicaba. Para un análisis sobre el proceso de transformación de la renta rural en renta urbana, consultar Pérez-Sáinz, 2012.
} 
En mutua imbricación, se inició con un acelerado fenómeno de transformación que, entre otras cosas, supuso la introducción de toda una nueva lógica residencialy comercial fundada en las tendencias hacia el "encerramiento" de los principales ámbitos de la vida cotidiana -i.e. trabajo, residencia, consumo, etc.- en espacios cerrados altamente controlados. Este confinamiento trajo consigo la constitución de un sentido común dominante basado en el "miedo" expresado como una constante sensación de (in) seguridad en la ciudad, el cual se fijó como uno de los discursos dominantes que ha guiado (y guía) la reestructuración urbana en la actualidad (Davis, 2003).

En el caso costarricense, las últimas décadas han sido un tiempo de incremento sostenido de la desigualdad social y de estancamiento de los niveles de pobreza y extrema pobreza, lo cual ha mostrado la (in)capacidad estatal para generar políticas públicas que cierren la brecha entre "ricos" y "pobres", la cual se ha ensanchado de la mano de los cambios en la estructura económica. En el espacio urbano, esto se ha expresado de una manera visible en la tendencia hacia la fragmentación socioespacial mediante la constitución de "islotes" de riqueza y de pobreza y la producción de imaginarios urbanos que otorgan distintos valores simbólicos a los lugares y a las gentes que habitan en ellos (Araya, 2010).

Más allá de tratarse de una distinción aparente, pues en realidad se trata de una realidad socio-espacial compleja y mutuamente imbricada, esta lógica de ciudad basada en "islotes" ha supuesto la clasificación de los espacios en función de la condición social de sus habitantes y ha cargado socialmente -de manera positiva o negativa- dichos espacios ${ }^{9}$. Dicha clasificación produce como un correlato necesario los discursos sobre la seguridad-inseguridad urbana.

La ciudad, en tanto espacio abierto, se ha posicionado desde dicho discurso como la antítesis de la seguridad, mientras los nuevos espacios cerrados en prácticamente una panacea urbana ${ }^{10}$. El neoliberalismo, en tanto discurso fuerte (Bourdieu, 1998), ha posicionado la cuestión de la seguridad-inseguridad como un poderoso mecanismo

\footnotetext{
8 En este punto, es importante indicar que se parte de la consideración de que son "los productores" los principales actores en los procesos de cambio urbano; si bien reconociendo la interdependencia dialéctica entre la producción y el consumo, esto implica fijar la producción como punto de partida para entender la "nueva geografía urbana" generada por el capital, en lugar de centrar la mirada en el consumo individualizado e interpretar fenómenos como la suburbanización exclusivamente desde el cambio en los patrones de consumo de los individuos.

De acuerdo con Araya (2010), la imagen de ciudad polarizada es uno de los seis imaginarios urbanos que se ciñen sobre la ciudad de San José. Esta supone la referencia a una fuerza centrífuga que busca generar un movimiento desde el centro urbano hacia la periferia (o suburbios). La nueva polarización dominante Oeste-Este/Centro/Norte-Sur plantea el Oeste-Este como "nuevos lugares de desarrollo" produciéndose un "complejo sistema simbólico de la ciudad que se caracteriza porque se sobrevalora la región Oeste y Este y se desvalorizan los antiguos sitios de población ubicados en el Norte, en el Sur y en el Oeste y Este más cercanos..." (p. 3).

10 Esto, por supuesto, desde el terreno de los discursos sobre el espacio, pues tanto en Costa Rica como en otros lugares se ha mostrado que los espacios cerrados no garantizan la seguridad, registrándose episodios violentos que incluso han supuesto la muerte de habitantes de urbanizaciones cerradas.
} 
ideológico para justificar el urbanismo neoliberall11 y, como señala Janoschka (2011), reproducir su propia geografía mediante la institucionalización de "...diferentes formas de "supuestas" políticas de prevención que incluyen la vigilancia (...) el nuevo diseño urbano y el control social" (pp. 124-125, Davis, 2003)12.

Sí, como sostiene Reguillo (2008), "a cada miedo (...) corresponden determinadas respuestas" (p. 71), el espacio urbano se ha transformado, desde los discursos basados en el miedo, en territorio "de nadie" o, lo que es lo mismo, en territorio de esa "alteridad amenazante" (p. 66) construida como el súmmum de todos los males de la ciudad. El miedo se instaura como uno de los determinantes de las nuevas formas de sociabilidad urbana que se configura desde la sospecha constante sobre los espacios, los tiempos y las personas. Para cada espacio existen determinados marcadores sociales, económicos y culturales que le asignan, como se señaló, una valoración, sea positiva o negativa, que en el mismo movimiento califica a las personas que habitan en dichos lugares. Si bien, la ciudad se conforma de un conjunto plural de espacios y, por lo tanto, la construcción social de miedo (Reguillo, 2008) se manifiesta de modo complejo según el lugar desde el que se enuncian los discursos sobre este; el centro de esta y sus márgenes inmediatas es concebido como un contenedor de desorden socio-espacial.

Ahora bien, tal como la literatura sobre urbanizaciones cerradas ha mostrado, en particular los trabajos de Svampa (2008), el encerramiento como práctica de clausura socio-espacial también supone una estrategia de distinción, en el sentidobourdieusiano; una forma de inscribir espacialmente la distancia social existente entre las distintas clases sociales, mediante la producción de espacios altamente homogéneos que de la misma manera funcionan como mecanismos, tanto materiales como simbólicos, de contención de la alteridad amenazante.

Dicha homogeneidad socio-espacial genera la sensación de encontrarse "entre iguales" (Alvarado y Jiménez, 2013) definiendo pautas de sociabilidad de clase según las cuales la exclusión física de la diferencia anula la posibilidad de los contactos in-deseados ${ }^{13}$.

\footnotetext{
11 En una conferencia pronunciada en 1998 en la Asamblea General de la FLACSO en la Ciudad de México, el politólogo germano-chileno, Norbert Lechner, señaló la emergencia de un estado de "malestar social difuso" surgido en el proceso de lo que él llamó "modernización" de los estados latinoamericanos desde los años ochenta. De acuerdo con Lechner, dicho malestar social instalado en la subjetividad de la gente encontraba en los "miedos" una de sus expresiones más complejas; fenómeno montado sobre el trípode: miedo al otro, miedo a la exclusión social y económica y miedo al sinsentido (1998, 179-181).

$12 \quad$ Para un análisis sobre cómo el miedo incide en las prácticas y percepciones sobre el espacio urbano de una población estigmatizada de San José - las trabajadoras sexuales- consultar Rojas (2014).

13 La homogeneidad socio-espacial no es un fenómeno exclusivo de las urbanizaciones cerradas ni tampoco de los sectores de clase media-alta y alta. Se necesitaría un estudio más detallado para identificar las diferentes expresiones de esta en el espacio urbano costarricense, no obstante cabe señalar que en el caso de los condominios y de los residentes de estos, una de los elementos distintivos es el carácter voluntario de su "exclusión" (o auto-exclusión) y por tanto la búsqueda de homogeneidad se transforma en un deseo de distinción.
} 


\section{Para estudiar las urbanizaciones cerradas}

En términos metodológicos, este estudio ha hecho uso de las técnicas cualitativas de investigación social. Para esto se desarrollaron un conjunto de entrevistas entre 2012 y 2013 a residentes de urbanizaciones cerradas en el distrito de San Rafael de Escazú. De la misma manera se entrevistó a habitantes de los barrios cercanos a estos desarrollos inmobiliarios, también a funcionarios municipales y a otros actores directamente ligados con los mismos ${ }^{14}$.

Hemos tomado como base el estudio de Thuillier (2005) en el cual propone estudiar este tipo de desarrollo desde sus implicaciones en la transformación de la urbanidad, definida esta como "...el conjunto de propiedades de un espacio urbano y las relaciones que establecen sus residentes con este espacio" (Thuillier, 2005, 6). Dichas propiedades se hacen manifiestas en las formas urbanas de un espacio urbano, en el conjunto de funciones urbanas y, finalmente, en las relaciones sociales que se desarrollan entre los habitantes de dicho espacio.

La forma urbana es definida como la materialidad de un espacio, como el conjunto de características físicas que hacen a su composición en cuanto tal. Dentro de esta se considera el tipo de construcción de las urbanizaciones cerradas y de las casas en su interior. También se incluye su ubicación en el espacio de la ciudad, en este caso San Rafael de Escazú. Las llamadas funciones urbanas hacen referencia a los usos que los habitantes de estos desarrollos hacen de los mismos y de otros espacios en el exterior de las urbanizaciones cerradas. Finalmente, las relaciones sociales constituyen el conjunto de interacciones individuales y colectivas entre habitantes de estos desarrollos entre sí y más allá de los muros.

Para cada una de estas tres categorías de análisis se hace una distinción entre "afuera" y "adentro" con el fin de diferenciar las características identificables tanto intra- como extra-muros. Esto posibilita un análisis no solo de lo que sucede en el interior de las urbanizaciones cerradas sino de cómo estas interactúan con su entorno cercano. No obstante, el "afuera" y el "adentro" no se consideran como dos realidades socio-espaciales independientes entre sí, sino como un proceso interconectado de intercambios, en el sentido social, entre ambas.

Cuando ha sido posible se ha incorporado información sobre las urbanizaciones cerradas generada de fuentes oficiales -estatales-, no obstante cabe señalar que ni en el

14 Se entrevistó a dos arquitectos cuyos trabajos recientes se han centrado en desarrollos inmobiliarios cerrados. 
nivel nacional (e.g. Instituto Nacional de Estadística y Censo, Ministerio de Vivienda y Urbanismo) niellocal(Municipalidad de Escazú) se ha hecho un seguimiento sistemático de la evolución de estas, con lo cual no se cuenta con registros sobre la cantidad, el tamaño, la extensión ni la cantidad de habitantes de estos desarrollos inmobiliarios ${ }^{15}$. La misma falta de conocimiento arrojó la consulta bibliográfica de estudios sobre la temática, evidenciando que en el caso de Costa Rica no existen investigaciones dedicadas a tratar de entender el fenómeno ni sus implicaciones socio-espaciales.

Por último, cabe señalar que en las secciones subsiguientes, particularmente las dedicadas a las categorías de análisis recién mencionadas se ha tomado como base para el análisis los testimonios de las personas entrevistadas tanto en el "adentro" como en el "afuera". Pese a esto, tratándose de un fenómeno escasamente pensado y hasta cierto punto naturalizado por las mismas personas, el trabajo con la teoría ha resultado fundamental para la interpretación de la información. Remitimos a las personas interesadas en conocer los testimonios a la investigación base de este trabajo (Alvarado y Jiménez, 2013, especialmente pp. 93-156).

\section{Escazú como ciudad "marca"}

Tal como señalamos recién, hacia la década de los años 1990 el sistema urbano costarricense sufrió un conjunto de transformaciones socio-espaciales; la formación de una ciudad crecientemente polarizada fue una de esas y, en palabras de Araya (2010), la constitución de una nueva polaridad Oeste-Este/Centro/Norte-Sur con los consiguientes imaginarios urbanos.

En esta nueva polaridad, el Oeste-Este se transformó en el "nuevo lugar de desarrollo" (Araya, 2010), la nueva dirección de éxito dentro de la cual Escazú, ubicada hacia el Oeste de San José, supuso un referente de este nuevo sistema urbano. En 2010, el ex alcalde de Escazú, Marco Segura, declaraba a un medio de comunicación escrita que el cantón representaba un "ícono" en el escenario urbano costarricense, mediante su especialización en la economía de servicios ${ }^{17}$.

Y es que tras el debilitamiento de la economía cafetalera en todo el país, incluyendo Escazú, se crearon las condiciones para la compra-venta de estas tierras y la constitución

\footnotetext{
15 Esto último considerando el momento de cierre de trabajo de campo en 2013.

16 En esta sección se presenta la información considerada más importante en relación con el cambio socio-espacial en Escazú. La misma se trata con más detalle en Alvarado y Jiménez, 2013, 48-60 y 94-100.

17 Marco Antonio Segura entrevista con La Nación, 23 de junio de 2010.
} 
de un dinámico mercado que transformó la renta agraria en renta urbana (Pérez Sáinz et al, 2012). Pérez Sáinz et al ha indicado que Escazú representó uno de los polos de modernización de la estructura urbana, tras el proceso mencionado. De acuerdo con él, en el caso de este cantón, como sucedió también en otros, la pérdida de importancia de las plantaciones de café posibilitó la compra-venta de considerables porciones de tierra que luego se reorientarían para el desarrollo inmobiliario de tipo residencial y comercial. Escazú, dice este autor, forma parte de uno de los cinco patrones identificados en el proceso de transformación de la renta agraria en renta urbana en el cual, hasta mediados de los años ochenta, una cuarta parte de la tierra se componía de cafetales, lo cual cambiaría durante la década siguiente con una reducción drástica de dicha proporción combinada con el acelerado crecimiento de un mercado inmobiliario (pp. 5-6).

Lo anterior se deduce de la información sobre la construcción de vivienda desde los años 1990, período durante el cual se registra un notorio crecimiento en la inversión en vivienda y en la cantidad de metros de construcción. En términos de la inversión en vivienda (en miles de millones) se pasó de ç2.478.030,00 en 1993 a ç8.518.122,89 en 2007, un aumento de $312 \%$.

En relación con la cantidad de urbanizaciones cerradas en el cantón de Escazú, utilizando el Sistema de Información Geoespacial (SIG) de la Municipalidad de Escazú, se trató de determinar el número de urbanizaciones cerradas en total y en los tres distritos que conforman el cantón. En total, en el cantón se contabilizaron 229 de este tipo de desarrollos inmobiliarios, mientras en la división por distritos, se contabilizaron $183(80 \%)$ en San Rafael, 30 (13\%) en San Miguel de Escazú y 16 (7\%) en San Antonio ${ }^{18}$. Junto con esto, el crecimiento exponencial del sector terciario (comercio y servicios) en el cantón de Escazú, constata las transformaciones acontecidas durante las últimas décadas. De acuerdo con datos de la Municipalidad de Escazú, este es el sector económico más dinámico de los últimos años, principalmente en el distrito de San Rafael que cuenta con el mayor número de licencias otorgadas para el establecimiento de comercios, pasando de 173 licencias en 1995 a 1.161 licencias en 2005, absorbiendo el 65,9\% del total de licencias activas durante ese año. Otro dato importante es el de la distribución relativa del territorio según los usos establecidos en el Plan Regulador, en el que se observa que las zonas residencial y comercial concentran el 46,41\% del total. Demanera progresiva, laurbanización deEscazú, antes caracterizado porla permanencia de una economía y una cultura de corte tradicional, se hizo de la mano de la acción 
privada de inversores, desarrolladores y promotores inmobiliarios que imprimieron su sello en el espacio. En 1992, el centro comercial Multiplaza Oeste fue inaugurado y esto representó una suerte de "punto cero" de esta nueva dinámica, que se tornaría dominante durante los 1990 y buena parte de los 2000.

Una última cuestión de suma importancia es la de enmarcar Escazú en su complejidad. Dicho cantón está compuesto por tres distritos -San Miguel Escazú, San Antonio, San Rafael- cada uno de los cuales comporta una dinámica socio-espacial distinta. Escazú, distrito uno, se posiciona como un espacio "bisagra", donde confluyen las distintas formas urbanas, tanto de carácter urbano como rural o semi-rural. En el caso de San Antonio, distrito dos, es donde más se nota la persistencia de las dinámicas de carácter rural, siendo en este donde se reproducen formas tradicionales tanto económicas -la siembra- como culturales. Mientras en San Rafael, distrito tres, es donde se evidencia con más claridad los cambios escenificados en el cantón durante las últimas décadas. Dicho distrito ha seguido un proceso de urbanización de la mano de la creciente oferta de desarrollos inmobiliarios tanto residenciales como comerciales y de la migración de sectores de clase media-alta y alta.

\section{El muro y la forma urbana}

\section{El intra-muros}

El muro, o los muros, en tanto materialidad son una manifestación de esa fractura socioespacial que ha supuesto una tendencia hacia el ensanchamiento de las desigualdades sociales. Preguntarse por estos no supone un fin en sí mismo; por lo menos no en el caso de este estudio, pues lo que interesa es tratar de establecer cómo los muros condicionan las formas de relacionarnos cotidianamente, cómo estos expresan una determinada forma de enfrentarse a los cambios en lo social, lo económico, lo político y lo cultural.

Tal como distintos autores han establecido (Thuillier, 2005; Svampa, 2008), los muros son la concreción espacial de la creciente distancia social entre los individuos, grupos sociales y clases sociales. En este sentido, los muros inscriben en el espacio, como en el caso de Escazú, la predominante polarización social.

Hacia adentro, los muros representan la posibilidad de auto-excluirse, esa condición que como señalaba Bauman es tan distinta de la exclusión en cuanto lo que marca es la 
intención de tomar distancia de la otredad. Los muros marcan la posibilidad de ejercer el control socio-espacial, un control que como se ha señalado antes se funda en un conjunto de binarismos contrastantes.

Dichos binarismos que participan en la producción social de espacio distinguen entre el "adentro" como lugar seguro-ordenado-controlado-previsible y el "afuera" como todo lo contrario: como lugar inseguro-desordenado-incontrolado-imprevisible. Dicho par, jerarquiza los espacios, designa para cada uno una valoración y lo mismo para quienes viven en ellos.

De esta manera, en el discurso el "adentro" se transforma en un espacio autosuficiente y que se dota de sus propias regulaciones, limitando la acción de los agentes extra-muros ${ }^{19}$. Si bien la legislación sobre los condominios, establece como mandato la formación de una organización de condóminos que se encargue de manejar lo relacionado a estos, esta no los faculta para limitar las funciones de las instituciones públicas en el interior de los condominios.

Junto con este control socio-espacial (o detrás de él) está la pretensión de mantener, en la medida de lo posible, una alta homogeneidad, principalmente de clase, en el intramuros. Claro está, dicho interés no es único de los condominios ni llega con estos, pues la construcción de los viejos barrios de clase en el centro de San José, como Barrio Amón, Barrio Escalante u otros, expresaba un deseo similar desde el siglo XIX. No obstante, dichos barrios no limitaban la continuidad socio-espacial y se inscribían dentro de un conjunto urbano más amplio en torno de la ciudad. En cambio, los condominios desde su misma constitución buscan constreñir la circulación como una forma de contener el contacto con la diferencia.

\section{El extra-muros}

Como recién se indicó, las urbanizaciones cerradas implican un corte en la continuidad de los espacios, marcan un límite tanto material como no-material, de los lugares por los que se puede circular y los que no se puede circular. Esta discontinuidad representa un cambio de considerable importancia, incluso si se considera que antes de estas en Escazú predominaban los predios cafetaleros y de otros tipos de producción que si bien

\footnotetext{
19 Entre otras cosas, han sido documentadas las dificultades de oficiales de la Fuerza Pública para ingresar en los condominios. Lo mismo ha sucedido con los encuestadores del Instituto Nacional de Estadística y Censos cuando les ha tocado desarrollar sus funciones en estos lugares.
} 
también eran de propiedad privada se constituían en muchos casos como espacios donde transcurrían ciertas dinámicas de la vida cotidiana ${ }^{20}$.

Junto con este "corte" en el espacio, sin duda una de las características más marcadas de la presencia de esta forma urbana en Escazú, como en otros lugares, es lo que se ha denominado como la visibilización-invisibilización de la distancia social o la paradoja socio-espacial (Thuillier, 2005; Alvarado y Jiménez, 2013). Estos espacios, mediante la instauración de mecanismos de control socio-espacial como el muro pero también la video-vigilancia perimetral y la presencia de seguridad privada, buscan el ocultamiento de lo que está-detrás-de-los muros; sin embargo, dicha ocultación también produce un efecto contrario: su evidenciamiento.

En principio, resulta casi imposible, más allá de la naturalización, no percatarse de la presencia de estos espacios cuando se transita por las calles de Escazú, en particular de San Rafael. De manera constante, el muro se manifiesta como el límite entre cada espacio.

Este límiteproducela sensacióndedistancia entrelosespaciosydelimita simbólicamente el lugar de las gentes en la estructura social. La homogeneidad socio-espacial creada hacia "adentro" no necesariamente se corresponde con la homogeneidad hacia "afuera" pues el muro posibilita que la diferencia "esté-más-cerca" sin mantener contacto con el intra-muros.

\section{Muros y funciones urbanas}

\section{El intra-muros}

En Costa Rica, y particularmente en Escazú, las urbanizaciones cerradas mantienen fundamentalmente una función residencial, y esto hace que necesariamente las otras funciones de la vida cotidiana de sus habitantes se descarguen más allá de los muros. Dicha mono-funcionalidad configura un tipo de espacio donde las prácticas urbanas son limitadas, en muchos casos, a la función de dormitorio mientras el trabajo, el estudio, el consumo y el ocio permiten mantener el contacto con el exterior. En este sentido, los binarismos que, como señalamos en la sección anterior, ordenan la lógica adentro-afuera operan más en el terreno de los discursos que en el de las prácticas sociales, en tanto no se trata de comunidades totales.

\footnotetext{
20 Dadas las limitaciones de espacio no se puede profundizar en este tema ni en otros, sin embargo cabe señalar que para los barrios cercanos a los cafetales, estos constituían un espacio donde transcurrían ciertos momentos de la vida cotidiana, incluyendo el juego para niños, niñas y jóvenes. Esto también fue indicado para el caso de Escazú y se puede consultar en Alvarado y Jiménez (2013).
} 
Ahora bien, si cambiamos de escala desde la unidad residencial que conforma el condominio hacia la configuración socio-espacial de todo el distrito de San Rafael, entonces nos encontramos con la constitución de un espacio urbano que pretende uniformar dichas funciones urbanas en torno de esa unidad residencial. De esta manera, junto con las urbanizaciones cerradas en este distrito se ha desarrollado toda una oferta (y una demanda, por supuesto) relacionada con las pautas de consumo y de sociabilidad de los sectores de clase media-alta y alta que la habitan.

Ese imaginario social que ha hecho de Escazú una "marca", un polo de "desarrollo", la conexión de Costa Rica con las tendencias mundiales, se corresponde con la puesta en escena de un conjunto de desarrollos de distinto tipo. Entre estos, los centros comerciales o malles han sido otra de las formas urbanas que marcan la transformación de este cantón desde que en 1992 se inaugurara la primera etapa de Multiplaza Oeste. Lo mismo con los complejos, también cerrados, de sistemas de educación que incluyen desde el maternal hasta la primaria y la secundaria, y también una forma un tanto más reducida de universidades privadas.

De este modo, si bien la mono-funcionalidad de las urbanizaciones cerradas hace que sus habitantes mantengan contactos constantes con el extra-muros, este extra-muros pareciera situarse como un adentro-en-el-afuera, como un conjunto interconectado de espacios cerrados que reproducen la distancia y la distinción social que tanto buscan estos sectores.

Volviendo sobre el intra-muros, se denota un infra-uso de los espacios comunes, los cuales son también parte de las estipulaciones de la legislación sobre el tema. Dichos espacios pensados en principio como puntos de encuentro entre los habitantes de las urbanizaciones cerradas, parecen no cumplir su función, mientras se denota una esperada ausencia de espacios públicos. El espacio es común en tanto plantea la posibilidad de hacer un uso compartido de las instalaciones, sea un rancho, un gimnasio o una cancha de tennis (o hasta de golf en casos más excepcionales), pero, más allá de esto, lo que se encuentra es un uso familiar de los mismos por lo que los encuentros entre los mismos habitantes son escasos, mostrando la debilidad constitutiva de ese "nosotros" tan promocionado por las desarrolladoras y las promotoras inmobiliarias. Otro elemento que hace a las funciones urbanas, yen estas a la forma cómo los habitantes de los condominios se relacionan con el extra-muros, es el uso de transporte privado -el automóvil-. Esto, tampoco reducible solo a estos habitantes ni consecuencia directa de este tipo de forma urbana, hace que la experiencia urbana transcurra, como recién se mencionó, entre espacios privados desde la residencia hacia la escuela, el colegio o la 
universidad, hacia el centro comercial o hacia la oficina, constriñendo las posibilidades incluso de esos contactos intermitentes con la otredad.

\section{El extra-muros}

Si en términos de su morfología, Escazú y en concreto San Rafael sufrió una profunda transformación de la mano de los condominios y otros desarrollos, lo mismo sucedió en el caso de las funciones urbanas para los "viejos" habitantes de este distrito.

Fuera de los muros, las posibilidades de encuentro entre los habitantes resultan bastante limitadas dada la casi total ausencia de espacios públicos y la tendencia hacia la privatización de los lugares de interacción social. Los centros comerciales, por ejemplo, se han constituido como uno, sino el principal, espacio de relacionamiento entre las personas, pero estos no son pensados como espacios inclusivos-incluyentes sino como exclusivos-excluyentes; sea de manera directa o indirecta, estos generan la sensación de pertenencia o no-pertenencia definida fundamentalmente por la capacidad de consumo transformada en una condición necesaria para sentir in-cluido en el mundo contemporáneo. De esta manera, otros espacios de interacción han tendido hacia su desaparición, es el caso de las ventas minoristas tipo pulperías u otras similares.

Cosa similar sucede con las posibilidades de circulación vial-peatonal en un espacio conformado para el vehículo privado. En San Rafael, cruzar una calle se ha transformado en un tema sensible dado el constante flujo vehicular en sus calles y la limitada disposición de mecanismos para posibilitar la circulación peatonal, como semáforos, cruces, aceras, etc.

En términos de la seguridad sucede una situación similar, en tanto la constante presencia de la seguridad privada en la entrada de todos los espacios privados contrasta con la escasa presencia de la Fuerza Pública en las calles de la zona. De esta manera, la seguridad se transforma en un bien que fluctúa en función de la posesión de recursos económicos. Y, como sucede en otros casos como el estado de calles, alcantarillas y zonas comunes, la presencia público-institucional depende de la distancia respecto de las urbanizaciones cerradas. 


\section{Muros y relaciones sociales}

\section{El intra-muros}

Las urbanizaciones cerradas, en tanto forma urbana, suponen también un conjunto de cambios en las relaciones sociales y en las formas de sociabilidad en el intra-muros y en el extra-muros. Hacia "adentro" el espacio está dispuesto conforme una lógica de control socio-espacial que posibilite la reproducción de vínculos "entre iguales". Si bien, no se trata de comunidades totales (Ascher citado en Svampa, 2008) las urbanizaciones cerradas proponen a sus residentes una forma de sociabilidad basada en la "familiaridad". Una "sociabilidad entre nos" (Svampa, 2008) donde se constituya un nosotros desde la homogeneidad socio-espacial que permite mantener la otredad fuera de los límites de los muros perimetrales.

La formación de este nosotros en el intra-muros se desarrolla de la mano de los discursos sobre la seguridad-inseguridad, haciendo de "adentro" el espacio para mantenerse seguro $^{21}$. En este sentido, es fundamental considerar que las urbanizaciones cerradas no solo niegan la ciudad en tanto forma urbana, sino sobre todo rechazan el tipo de relaciones sociales que en esta transcurren y que están marcadas por el carácter noanticipante de las mismas. La ciudad como espacio potencial de mutua identificación entre los distintos individuos, grupos y clases sociales se reemplaza por un espacio donde se ejerce un "control disciplinario de las relaciones sociales" (Méndez citado en Rodríguez, 2004, 54) 22. De la misma manera, en la conformación de este "nosotros" resulta fundamental la producción de estrategias de distinción social; "el éxito tiene donde vivir" como promocionaba una de las publicaciones de los desarrolladores inmobiliarios en Costa Rica, le habla a los sectores de clase media-alta y alta y "flirtea" con sus deseos de diferenciarse ${ }^{23}$.

\footnotetext{
21 En una sugerente formulación, Lechner (1998) planteaba que "el miedo a los otros es tanto más fuerte cuanto más frágil es el 'nosotros"' (p. 183, énfasis nuestro). Esta idea que necesitaría de más desarrollo, insinúa la posibilidad de encontrar una relación entre el miedo y la tendencia hacia la constitución de relaciones sociales de baja intensidad, no solo en el intra-muros. Asimismo, cabe preguntarse si el miedo a los otros no funciona como uno de los factores de cohesión social entre los residentes de las urbanizaciones cerradas.

22 Es necesario introducir un conjunto de matices sobre esta cuestión. En primer lugar, no se trata de hacer de la ciudad un fetiche, pensando que por sí misma garantiza el reconocimiento de la diversidad social. No obstante, la disposición espacial de esta posibilita, como potencialidad, el encuentro entre diferentes y supone un cierto grado de incertidumbre sobre el tipo de contactos en la vida cotidiana. En segundo lugar, como se señaló en una sección anterior, el control socio-espacial es una de las características dominantes en el actual proceso de (re)estructuración urbana seguido por la mayoría de ciudades en el mundo y, en este sentido, también se ejerce un "control disciplinario" sobre la ciudad por parte de actores públicos y privados.

23 Haría falta un estudio para ocuparse de la centralidad que tienen los desarrolladores y los promotores inmobiliarios en la fabricación de estos discursos. No obstante, la revisión de sus publicaciones periódicas permite plantear que muchas de las ideas dominantes sobre la relación entre las urbanizaciones cerradas y la seguridad o el mismo tema de la homogeneidad socio-espacial, son "filtradas" mediante estas. Asimismo, en la constitución de un "nosotros" es fundamental la promoción inmobiliaria, pues se trata de crear un nosotros ficticio incluso antes de que los futuros residentes se conozcan entre sí.
} 
Pero, más allá de estos discursos, la dinámica en el intramuros, para el caso de San Rafael de Escazú pero también para otros casos como lo ha mostrado la bibliografía sobre el tema, habla de un constreñimiento de los intercambios sociales entre sus residentes. Predominan relaciones sociales "de baja intensidad", pese a que desde el discurso se erige la idea de retorno a la comunidad, a la vida de barrio ${ }^{24}$. Las pautas de sociabilidad se circunscriben a encuentros ocasionales en los espacios comunes, interacciones no-pactadas.

\section{El extra-muros}

En tanto no son comunidades totales, las urbanizaciones cerradas y sus habitantes necesariamente descargan un conjunto de funciones sobre la ciudad, sean estas el trabajo, el estudio, el consumo, etc. No obstante, si se amplía la escala desde el condominio como unidad hacia el distrito de San Rafael de Escazú, es posible plantear que en el entorno se ha configurado todo un circuito socio-espacial que limite en la medida de lo posible el intercambio con la diferencia.

En términos de las relaciones sociales entre el "adentro" y el "afuera", los residentes de las urbanizaciones cerradas mantienen una buena cantidad de sus interacciones cotidianas con gente más allá de los muros. Dichos intercambios, como es esperable, son de distinta naturaleza, pero en términos de su entorno inmediato son prácticamente nulos. El extra-muros cercano y la gente que habita en los lugares aledaños a los condominios desconocen casi en su totalidad a sus "vecinos". La edificación de estos, supuso entre otras cosas una serie de cambios en las formas de sociabilidad de los habitantes históricos de San Rafael.

Hasta antes de los años 1990, cuando se inicia la construcción del Centro Comercial Multiplaza, la dinámica social, económica y cultural de Escazú giraba alrededor de su ruralidad. De hecho, dicho cantón se reconocía como uno de los "contenedores" de las tradiciones costarricenses como es el caso de los boyeros. No obstante, el proceso de cambio hacia la condición rural-urbano (Thuillier, 2005) representó el desmontaje de los patrones de sociabilidad propios y su sustitución por unos de tipo urbano y de menor familiaridad debido a la llegada de gentes de otros lugares y con otras formas de relacionarse. En este sentido, para los mismos habitantes de Escazú los condominios supusieron un cambio fundamental en su cotidianeidad.

\footnotetext{
24 Los residentes de urbanizaciones cerradas entrevistados en nuestra investigación, expresaban la falta de interacciones sociales regulares con los residentes. En términos generales, estos se limitaban a encontrarse en los espacios en común como los garajes o las entradas/salidas de los condominios.
} 
Pese a esto, la propia disposición material de esta forma urbana impide el contacto entre el "adentro" y el "afuera" lo cual es expresado por los mismos habitantes de las zonas cercanas para quienes sus "vecinos" son casi unos desconocidos. Esto, sin embargo, no es tomado como un problema social que empobrece la vida cotidiana en el cantón, sino se interpreta como una situación de coexistencia espacial, donde la fragmentación es naturalizada.

De esta manera, el "adentro" y el "afuera" se configuran desde la cotidianeidad de Escazú como dos realidades socio-espaciales paralelas; las desigualdades sociales se inscriben espacialmente haciéndose manifiestas en la materialidad de este cantón y consolidando un modelo de ciudad suburbanizada.

\section{Conclusiones}

El proceso de neoliberalización de la estructura económica costarricense produjo un (esperado) conjunto de cambios en la ciudad. En el escenario de transformaciones mundiales de la dinámica urbana, de la mano de lo que Sassen (2004) ha llamado la "ciudad global", las distintas entidades urbanas y la multi-escalaridad expresada en estas, ha supuesto procesos de "conexión” y "desconexión” en relación con los contextos nacionales, regionales y mundiales.

El dominante urbanismo neoliberal de las últimas décadas ha posibilitado una creciente polarización socio-espacial bajo la cual se han ordenado los espacios de acuerdo con distintos criterios, pero siendo el económico fundamental. La persecución de altas tasas de ganancia ha guiado la (re)estructuración urbana en Costa Rica, como en prácticamente todo el mundo, y ha encontrado en la conjunción sui generis entre los actores públicos y los actores privados, un escenario propicio para modificar el sistema urbano.

En el caso de este artículo, el interés principal se centró en intentar mostrar cómo el proceso de de-concentración urbana o de suburbanización de la ciudad seguido durante este período supuso un movimiento desde el centro hacia la periferia ("los suburbios"), movimiento de gentes, pero sobre todo de capitales, posibilitando la expansión de la ciudad más allá de sus límites conocidos y la introducción de una serie de nuevas modalidades residenciales y comerciales.

Las urbanizaciones cerradas, como nueva modalidad residencial, han implicado cambios en la urbanidad, no solo de Escazú sino de toda la Gran Área Metropolitana, pues estas 
se han expandido en todas las direcciones. La modificación en las formas urbanas y en las funciones urbanas y los consiguientes cambios en las relaciones sociales en el intra-muros y en el extra-muros, han ocasionado una fragmentación socio-espacial visible en el espacio de este cantón.

En este sentido, el cantón de Escazú se transformó, en primer lugar, en sus formas urbanas, mediante la reconfiguración socio-espacial de una morfología predominantemente rural a una urbana donde, sin embargo, permaneció un tipo de "ruralismo bucólico" fundado en la presencia de lo "natural" para re-ligar el pasado rural con el presente urbano de la estructura socio-espacial en curso. De esta manera, el cantón se estableció, en el imaginario social, como el lugar para "habitar", la dirección de los "triunfadores", la marca desde donde se desarrolla la diferenciación social entre los distintos grupos de la estructura social.

Las transformaciones socio-espaciales relacionadas con la instalación de esta forma urbana en el cantón de Escazú y, en general, en otras direcciones de la ciudad costarricense, establece un debate sobre el tipo de urbanidad desarrollado durante las últimas tres décadas en el país. En este sentido, la noción de "urbanidad privada" 25 , posibilita entablar una discusión sobre las consecuencias de este proceso, más allá de la dinámica in situ de las urbanizaciones cerradas. Las urbanizaciones cerradas, inscritas en el tipo de "urbanidad privada", se desarrollan con base en criterios de obtención de ganancias orientados por el interés privado, particularmente de actores sociales como las promotoras y las desarrolladoras inmobiliarias, mientras se des-entiende de la cosa pública en términos del espacio urbano. El espacio producido, no es un espacio en función de lo colectivo, sino de lo individual, de lo privado; el uso de los espacios es un uso individual, privado. 


\section{Referencias}

Alvarado, A. y Jiménez, G. (2013). Urbanizaciones cerradas en Costa Rica: transformaciones socio-espaciales en la urbanidad y segregación socio-residencial en el distrito de San Rafael de Escazú (1990-2012). Tesis de Licenciatura. Costa Rica: Universidad de Costa Rica.

Araya, M. (2010). San José. De "París en miniatura" al malestar en la ciudad. Medios de comunicación e imaginarios urbanos. Costa Rica: EUNED.

Bourdieu, P. (2002). La esencia del neoliberalismo. En Bourdieu, P. Seis artículos de Pierre Bourdieu publicados en Le Monde Diplomatique. Santiago: Editorial Aún Creemos en los Sueños.

Chacón, L. y Zuñiga, J. (2014). "Más allá de las fronteras de la ciudad: la vida en el espacio de la exclusión". En Revista de Ciencias Sociales, 146, 127-139. Costa Rica: Universidad de Costa Rica.

Davis, M. (2003). Ciudad de cuarzo. España: Lengua de Trapo.

Harvey, D. (2007). Breve historia del neoliberalismo. España: Akal.

Janoschka, M. (2011). "Geografías urbanas en la era del neoliberalismo. Una conceptualización de la resistencia local a través de la participación y la ciudadanía urbana”. En Investigaciones Geográficas, Boletín del Instituto de Geografía, 76, 118132. México: Universidad Nacional Autónoma de México.

Janoschka, M. y Glasze, G. (2003). "Urbanizaciones cerradas: un modelo analítico". Revista Ciudades, 59, 9-20. México: Red de Investigación Urbana A.C.

Lechner, N. (1998). "Nuestros miedos". Revista Perfiles Latinoamericanos, 13, diciembre, 179-198. México: Facultad Latinoamericana de Ciencias Sociales.

Mora, S. (2007). "Costa Rica en la década de 1980: estrategias de negociación política en tiempos de crisis ¿Qué pasó después de la protesta?” Revista inter.c.a.mbio, 5, 165183. Costa Rica: Universidad de Costa Rica. 
Pérez Sáinz, J.Py Alvarado, K. (2012). Poder yacaparamiento de rentas en nuevos ejes de acumulación en Costa Rica. Ponencia presentada en las VII Jornadas de Investigación, Instituto de Investigaciones Sociales. Costa Rica: Universidad de Costa Rica.

Reguillo, R. (2008). "Sociabilidad, inseguridad y miedos. Una trilogía para pensar la ciudad contemporánea". Revista Alteridades, 18(36), julio-diciembre, 63-74. México: Universidad Autónoma Metropolitana Unidad Iztapalapa.

Rodríguez, I. (2004). "Urbanizaciones cerradas en Latinoamérica”. Revista Ería, 63, 53-57. España: Universidad de Oviedo.

Rojas, M. (2014). "Geografías del miedo de mujeres trabajadoras sexuales: entre habitares y percepciones". Revista de Ciencias Sociales, 145, 61-80. Costa Rica: Universidad de Costa Rica.

Sassen, S. (2004) . "La ciudad global: introducción a un concepto". En: BBVA (Ed.). Las múltiples caras de la globalización, p. 51-62. Madrid. BBVA.

Smith, N. (2005). El redimensionamiento de las ciudades: la globalización y el urbanismo neoliberal. En Harvey, David y Smith, Neil (eds.). Capital financiero, propiedad inmobiliaria y cultura. España: Museo de Arte Contemporáneo de Barcelona/ Universidad Autónoma de Barcelona.

Svampa, M. (2008). Los que ganaron. La vida en los countries y en los barrios privados. Argentina: Biblos.

Theodore, N., Peck, J. y Brenner, N. (2009). "Urbanismo neoliberal: la ciudad y el imperio de los mercados". Revista Temas Sociales, 66, marzo, 1-12. Chile: SUR Corporación de Estudios Sociales y Educación.

Thuillier, G. (2005). El impacto socio-espacial de las urbanizaciones cerradas: el caso de la Región Metropolitana de Buenos Aires. Revista EURE, 93, 5-20. Chile: Pontificia Universidad Católica de Chile. 
Este artículo forma parte de:

\section{REVISTARQUIS}

Para más información, artículos, e instructivo de publicación, visite:

www.arquis.ucr.ac.cr/revistarquis.html 